\title{
Многократное изменение электрон-фононного взаимодействия в квантовых ямах с диэлектрически различными барьерами
}

\author{
(C) А.Ю. Маслов, О.В. Прошина \\ Физико-технический институт им. А.Ф. Иофрфе Российской академии наук, \\ 194021 Санкт-Петербург, Россия \\ ฯ E-mail: proshina.ton@mail.ioffe.ru
}

Поступила в Редакцию 1 июля 2021 г.

В окончательной редакции 2 августа 2021 г.

Принята к публикации 9 сентября 2021 г.

\begin{abstract}
Теоретически исследованы особенности взаимодействия заряженных частиц с полярными оптическими фононами для квантовых ям с несимметричными по своим диэлектрическим свойствам барьерами. Показано, что в узких квантовых ямах наибольший вклад дает взаимодействие с интерфейсными фононными модами. Найдены параметры электрон-фононного взаимодействия при различных значениях фононных частот в материалах барьеров. Показано, что в подобных структурах возможно значительное (почти на порядок) изменение параметров электрон-фононного взаимодействия, что в принципе позволяет проследить переход от слабого взаимодействия к сильному в однотипных квантовых ямах с различным составом материалов барьеров. Найдены условия, при которых в несимметричной структуре возможно усиление электронфононного взаимодействия по сравнению с симметричной структурой, в которой в качестве барьеров использованы те же материалы.
\end{abstract}

Ключевые слова: квантовая яма, электрон-фононное взаимодействие, полярон, асимметричные барьеры.

DOI: $10.21883 /$ FTP.2022.01.51819.9705

\section{1. Введение}

Современная технология изготовления полупроводниковых наноструктур позволяет эффективно изменять фононные свойства таких объектов. В связи с этим в последнее время достаточно широко используется термин „инженерия фононных состояний“ $[1,2]$. Спектр фононных состояний критически важен для тепловых свойств наноструктур и играет важную роль в модификации электрических и оптических свойств.

В наноструктурах существенно изменяются также параметры электрон-фононного взаимодействия. Особый интерес представляет взаимодействие заряженных частиц с полярными оптическими фононами. Оценки показывают, что в симметричных квантовых ямах возможна реализация сильного электрон-фононного взаимодействия с образованием поляронов большого радиуса [3]. Для этого необходимо использовать квантовые ямы с барьерами, изготовленными из материалов с высокой степенью ионности. При этом ионность материала самой квантовой ямы особого значения не имеет. Насколько нам известно, экспериментально такие возбуждения пока не наблюдались. Значительного изменения характера электрон-фононного взаимодействия следует ожидать в квантовых ямах с несимметричными по своим диэлектрическим свойствам барьерами. Подобные структуры в последние годы исследуются экспериментально [4-6]. Кроме того, они могут входить в качестве составных элементов в многослойные структуры, которые используются для создания солнечных элементов [7-11]. Изменение характера электрон-фононного взаимодействия связано с тем, что в несимметричных структурах со- поставимый вклад во взаимодействие должны давать несколько ветвей фононного спектра. В случае симметричных структур, как правило, преобладающий вклад вносит только одна фононная мода.

В настоящей работе исследованы особенности взаимодействия заряженных частиц с оптическими фононами для квантовых ям с несимметричными по своим диэлектрическим свойствам барьерами. Для нахождения фононного спектра и определения особенностей электронфононного взаимодействия используется модель диэлектрического континуума. Этот подход позволяет адекватно описывать фононные свойства гетероструктур в тех случаях, когда все характерные параметры задачи с размерностью длины превышают постоянную решетки используемых материалов. Для структур, содержащих моноатомные слои, могут потребоваться более сложные и громоздкие теоретические модели. В рамках нашего подхода показано, что за счет изменения свойств барьеров можно изменять величину электрон-фононного взаимодействия в несколько раз. Найдены условия, при которых в несимметричной структуре можно реализовать усиление взаимодействия по сравнению с симметричной структурой с использованием того же материала барьеров.

\section{2. Спектр интерфейсных фононов}

Будем рассматривать трехслойную планарную структуру, состоящую из области квантовой ямы с диэлектрической проницаемостью $\varepsilon_{w}(\omega)$ и двух различных барьеров с проницаемостями $\varepsilon_{l}(\omega)$ и $\varepsilon_{r}(\omega)$. Индексами $l$ 
и $r$ обозначены диэлектрические проницаемости левого и правого барьеров соответственно. В области фононных частот все диэлектрические функции имеют одинаковый вид:

$$
\varepsilon_{k}(\omega)=\varepsilon_{\infty, k} \frac{\omega_{L O, k}^{2}-\omega^{2}}{\omega_{T O, k}^{2}-\omega^{2}}
$$

с различными значениями частот продольных $\omega_{L O, k}$ и поперечных $\omega_{T O, k}$ фононов. Здесь индекс $k$ соответствует $l, r$ или $w$. При диэлектрической проницаемости, определяемой выражением (1), для всех слоев выполняется соотношение Лиддена-Закса-Теллера:

$$
\varepsilon_{k}(0)=\varepsilon_{\infty, k} \frac{\omega_{L O, k}^{2}}{\omega_{T O, k}^{2}}
$$

Электронный спектр барьеров не имеет определяющего значения для нас. При рассмотрении различных барьеров существенным оказывается лишь то, что они во всех случаях должны приводить к локализации носителей в области квантовой ямы. Наибольший интерес для нас в дальнейшем будут представлять интерфейсные оптические фононы. Их спектр можно найти, используя стандартные граничные условия на двух границах квантовой ямы [12]. Для рассматриваемой структуры он определяется решением следуюшего уравнения:

$$
e^{-|\mathbf{q}| a} \frac{\varepsilon_{w}(\omega)-\varepsilon_{l}(\omega)}{\varepsilon_{w}(\omega)+\varepsilon_{l}(\omega)}=e^{|\mathbf{q}| a} \frac{\varepsilon_{w}(\omega)+\varepsilon_{r}(\omega)}{\varepsilon_{w}(\omega)-\varepsilon_{r}(\omega)}
$$

Здесь $a$ - ширина квантовой ямы, q - двумерный вектор фонона в плоскости ямы. В общем случае решение уравнения (3) содержит две ветви оптических фононов, локализованных вблизи левой границы квантовой ямы, и две ветви фононов, локализованных вблизи правой границы. Эти ветви взаимодействуют между собой, что приводит к достаточно сложной картине для электронфононного взаимодействия.

Спектр оптических фононов и характер электронфононного взаимодействия существенно упрощаются для узких квантовых ям. Обычно наибольший интерес представляет собой взаимодействие в области, протяженностью порядка радиуса поляронного состояния $r_{p}$, что соответствует значениям волнового вектора $q \approx r_{p}^{-1}$. При выполнении условия

$$
q a \ll 1 .
$$

которое обычно реализуется в полупроводниковых ямах шириной $L \sim 50 \AA$, уравнение (3) значительно упрощается. В старшем порядке по параметру (4) из него полностью выпадают диэлектрические свойства области квантовой ямы. В результате из (3) получим

$$
\varepsilon_{r}(\omega)+\varepsilon_{l}(\omega)=0 .
$$

Уравнение (5) формально совпадает с уравнением для определения спектра интерфейсных фононов на одиночной гетерогранице [12]. При наличии несимметричных барьеров с $\varepsilon_{r}(\omega) \neq \varepsilon_{l}(\omega)$ оно имеет два решения для интерфейсных фононов. Причем для нашей трехслойной структуры одно имеет максимум интенсивности на левой границе ямы, а другое на правой.

Две другие ветви, которые содержит точное уравнение (3), из приближенного уравнения (5) выпадают. Области существования решений уравнения (5) зависят от фононных спектров материалов барьеров. Если области фононных частот материалов левого и правого барьеров перекрываются, то важным оказывается порядок расположения этих частот. Пусть для определенности $\omega_{L O, l}<\omega_{L O, r}$, а $\omega_{T O, l}>\omega_{T O, r}$. Тогда частоты интерфейсных фононов попадают в следующие интервалы:

$$
\begin{aligned}
& \omega_{T O, r}<\omega_{1}<\omega_{T O, l} \\
& \omega_{L O, l}<\omega_{2}<\omega_{L O, r} .
\end{aligned}
$$

При изменении порядка следования фононных частот в барьерах естественным образом изменяются и интервалы (6).

При несовпадающих областях фононных частот барьеров частоты интерфейсных фононов лежат в интервалах

$$
\begin{gathered}
\omega_{T O, l}<\omega_{1}<\omega_{L O, l} \\
\omega_{T O, r}<\omega_{2}<\omega_{L O, r} .
\end{gathered}
$$

Два решения, соответствующие частотам

$$
\omega_{T O, w}<\omega_{3,4}<\omega_{L O, w},
$$

в рассматриваемом приближении не учитываются. Безусловно, уравнение (5) неточно описывает спектр интерфейсных фононов из интервалов (6) и (7) при $q a \geq 1$. Однако именно в области (4), как будет показано далее, найденные фононные ветви дают наибольший вклад в электрон-фононное взаимодействие.

\section{3. Электрон-фононное взаимодействие}

Следуя данным работы [12], получим выражение для гамильтониана электрон-фононного взаимодействия $H_{\text {int }}$ в рассматриваемой структуре. Его можно представить в таком же виде, как и в случае симметричной структуры [13]:

$$
\begin{aligned}
H_{\text {int }, i}= & \sum_{\mathbf{q}}\left(\frac{2 \pi \omega_{i} e^{2}}{L^{2}}\right)^{1 / 2} \frac{\exp (i \mathbf{q} \rho)}{\sqrt{2 q}} f_{i}(\mathbf{q}, z) F_{i}(q, \omega) \\
& \times\left(a_{i}(\mathbf{q})+a_{i}^{+}(\mathbf{q})\right),
\end{aligned}
$$

где $L^{2}-$ нормировочная площадь квантовой ямы, $a_{i}(\mathbf{q})$ и $a_{i}^{+}(\mathbf{q})$ - операторы уничтожения и рождения фононов ветви с номером $i$ (четыре ветви в полном спектре и две в области $q a \ll 1)$. Множитель $f_{i}(\mathbf{q}, z)$ описывает распределение интенсивности возбуждений по направлению, перпендикулярному плоскости квантовой ямы. 
В рассматриваемом случае внутри квантовой ямы при $|z| \leq a / 2$ он имеет вид

$$
f_{i}(\mathbf{q}, z)=\gamma_{i}\left(\omega_{i}\right) e^{q z}+\gamma_{i}^{-1}\left(\omega_{i}\right) e^{-q z},
$$

где параметр $\gamma_{i}$ определяется следующим выражением:

$$
\gamma_{i}\left(\omega_{i}\right)=e^{-q a} \frac{\varepsilon_{w}\left(\omega_{i}\right)-\varepsilon_{l}\left(\omega_{i}\right)}{\varepsilon_{w}\left(\omega_{i}\right)+\varepsilon_{l}\left(\omega_{i}\right)}=e^{q a} \frac{\varepsilon_{w}\left(\omega_{i}\right)+\varepsilon_{r}\left(\omega_{i}\right)}{\varepsilon_{w}\left(\omega_{i}\right)-\varepsilon_{r}\left(\omega_{i}\right)}
$$

Выражение (9) для $f_{i}(\mathbf{q}, z)$ отличается от аналогичных выражений для структур с симметричными барьерами тем, что в данном случае нельзя выделить симметричные и антисимметричные моды.

Наибольшее отличие от симметричных структур возникает для коэффициентов $F_{i}\left(q, \omega_{i}\right)$ :

$$
\begin{aligned}
& F_{i}^{-1}\left(q, \omega_{i}\right)=\left[\beta_{l}^{-1}\left(\omega_{i}\right)\left\{\gamma_{i}^{2}\left(\omega_{i}\right) e^{-q a}+2+\gamma_{i}^{-2}\left(\omega_{i}\right) e^{q a}\right\}\right. \\
& +\beta_{r}^{-1}\left(\omega_{i}\right)\left\{\gamma_{i}^{-2}\left(\omega_{i}\right) e^{-q a}+2+\gamma_{i}^{2}\left(\omega_{i}\right) e^{q a}\right\} \\
& \left.+\beta_{w}^{-1}\left(\omega_{i}\right)\left\{\left(e^{q a}-e^{-q a}\right)\left(\gamma_{i}^{2}\left(\omega_{i}\right)+\frac{1}{\gamma_{i}^{2}\left(\omega_{i}\right)}\right)+2 q a\right\}\right]^{-1 / 2} .
\end{aligned}
$$

Выражение (11) описывает взаимодействие заряженных частиц со всеми ветвями интерфейсных фононов. Здесь функция $\beta_{i}\left(\omega_{i}\right)$ имеет вид [12]

$$
\beta_{i}(\omega)=\left[\frac{1}{\varepsilon_{i, \infty}}-\frac{1}{\varepsilon_{i, 0}}\right] \frac{\omega_{L O, i}^{2}}{\omega^{2}}\left[\frac{\omega^{2}-\omega_{T O, i}^{2}}{\omega_{L O, i}^{2}-\omega_{T O, i}^{2}}\right]^{2}
$$

Из выражения (11) видно, что при $q a \ll 1$ основной вклад дает взаимодействие, содержащее параметры $\beta_{l}(\omega)$ и $\beta_{r}(\omega)$, которые определяются диэлектрическими свойствами материалов барьеров. Вклады, обусловленные свойствами материала квантовой ямы и содержащие функцию $\beta_{w}(\omega)$, возникают лишь в более высоких порядках по параметру (4). Выражения (9) и (11) значительно упрощаются при выполнении условия (4). В наибольшем порядке по параметру (4) постоянную электрон-фононного взаимодействия $\alpha_{k}$ (аналог константы Фрелиха для объемных материалов) можно представить в следующем виде:

$$
\alpha_{i}=e^{2}\left(\frac{m}{2 \omega_{i}}\right)^{1 / 2} R_{\mathrm{as}}\left(\omega_{i}\right)
$$

где $i=1,2, m-$ масса носителей в квантовой яме, а последний множитель $R_{\text {as }}$ имеет вид

$$
R_{\mathrm{as}}\left(\omega_{i}\right)=\left|F_{i}\left(q \omega_{i}\right) f_{i}(\mathbf{q}, z)\right|^{2} \cong \frac{2 \beta_{r}\left(\omega_{i}\right) \beta_{l}\left(\omega_{i}\right)}{\beta_{r}\left(\omega_{i}\right)+\beta_{l}\left(\omega_{i}\right)} .
$$

Выражение (14) означает, что в достаточно узких квантовых ямах энергию электрон-фононного взаимодействия можно представить в виде постоянной по ширине ямы величины. Эта величина соответствует поляризации, создаваемой барьерами. В достаточно узких квантовых ямах эта поляризация мало изменяется на ширине ямы. При этом различие в пространственном положении максимумов взаимодействия для $\omega_{1}$ и $\omega_{2}$ оказывается несущественным. Диэлектрические свойства материала квантовой ямы в данном приближении также оказываются несущественными. Выражение для параметра $R_{\text {as }}\left(\omega_{i}\right)$ следует сопоставлять с аналогичным выражением для $R_{\mathrm{sim}}\left(\omega_{i}\right)$, возникающим при нахождении константы электрон-фононного взаимодействия в симметричных квантовых ямах. Ранее было показано [3], что в симметричных структурах при выполнении условия (4) эта величина оказывается равной

$$
R_{\text {sim }}=\frac{1}{\varepsilon_{\mathrm{opt}}}=\frac{1}{\varepsilon_{\infty}}-\frac{1}{\varepsilon_{0}},
$$

где величины $\varepsilon_{\infty}$ и $\varepsilon_{0}$ относятся к материалу барьеров. При этом частота интерфейсной моды близка к частоте $\omega_{L O}$ продольных оптических фононов материала барьеров, что и приводит к выражению (15). Выражения $R_{\mathrm{sim}, r}\left(\omega_{i}\right)$ и $R_{\mathrm{sim}, l}\left(\omega_{i}\right)$ будут соответствовать квантовой яме, где в качестве обоих барьеров использован материал либо правого $(r)$, либо левого $(l)$ барьеров асимметричной структуры.

В случае асимметричной структуры с разными барьерами частота фононов будет несколько отличаться как от $\omega_{L O, l}$, так и от $\omega_{L O, r}$. Хоть это отличие достаточно мало, его важно учитывать для корректного нахождения параметров электрон-фононного взаимодействия. На рис. 1 и 2 представлены модельные расчеты для параметров $R_{\mathrm{as}}\left(\omega_{i}\right)$ при различных соотношениях фононных частот материалов барьеров. Рассмотрен случай, когда области фононных частот барьеров перекрываются. Линейная зависимость фононных частот от некоторого внешнего параметра может быть реализована при использовании в качестве барьеров твердых растворов различного

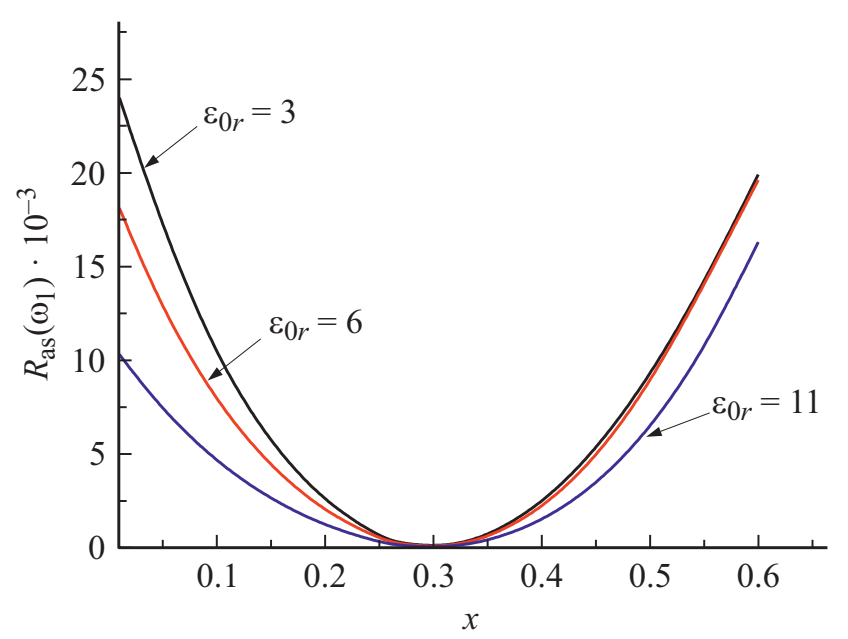

Рис. 1. Безразмерный параметр электрон-фононного взаимодействия $R_{\text {as }}\left(\omega_{1}\right)$ при изменении диэлектрических свойств барьеров. Использованы модельные зависимости для фононных частот барьеров $\omega_{L O l}=1.8 \omega_{T O l}, \omega_{T O r}(x)=(1.3-x) \omega_{T O l}$, $\omega_{L O r}(x)=(1.5+x) \omega_{T O l} ; \omega_{T O l}<\omega_{1}<\omega_{T O r} ; \varepsilon_{0 l}=4$. 


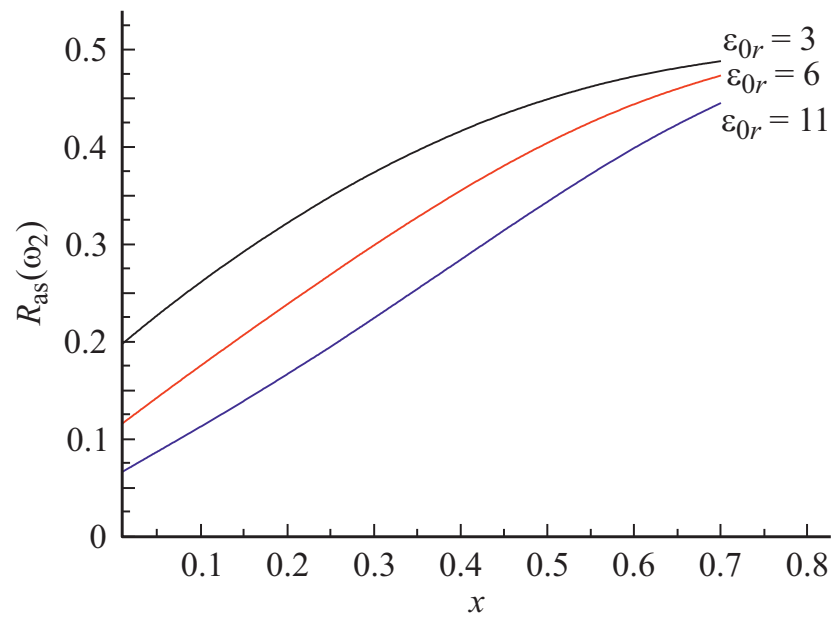

Рис. 2. Безразмерный параметр электрон-фононного взаимодействия $R_{\text {as }}\left(\omega_{2}\right)$ при изменении диэлектрических свойств барьеров. Использованы модельные зависимости для фононных частот барьеров $\omega_{L O l}=1.8 \omega_{T O l}, \omega_{T O r}(x)=(1.3-x) \omega_{T O l}$, $\omega_{L O r}(x)=(1.5+x) \omega_{T O l} ; \omega_{L O l}<\omega_{2}<\omega_{L O r} ; \varepsilon_{0 l}=4$.

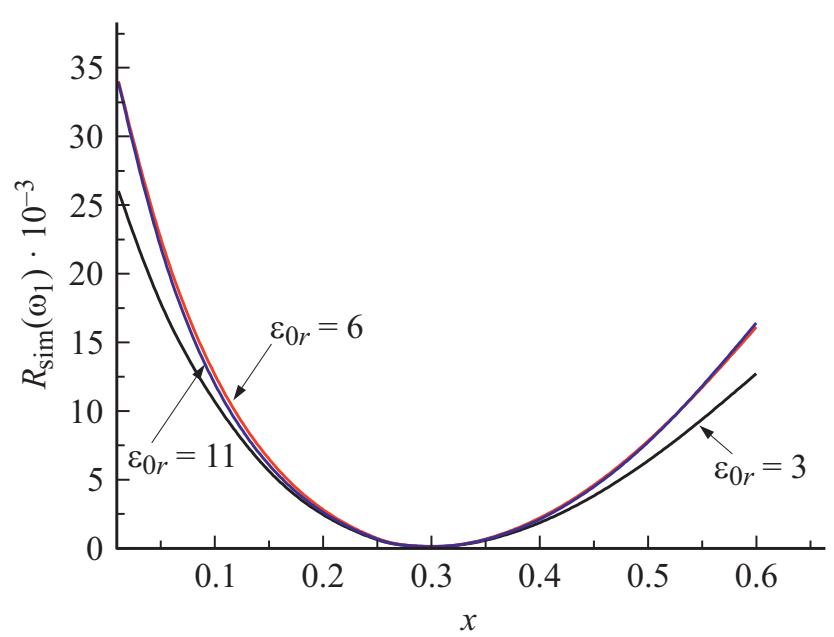

Рис. 3. Безразмерный параметр электрон-фононного взаимодействия $R_{\text {sim }}\left(\omega_{1}\right)$ для одинаковых барьеров сорта $r$ с теми же модельными зависимостями частот для правого барьера, что и на рис. 1 и 2.

состава. Из рис. 1 и 2 видно, что взаимодействие с одной из интерфейсных фононных мод оказывается на один-два порядка больше, чем с другой. Это связано с тем, что частота $\omega_{1}$ находится между двумя $T O$-модами частот барьеров и она по параметру электрон-фононного взаимодействия близка к аналогичным параметрам поперечных колебаний. На рис. 3 и 4 представлены значения безразмерного параметра электрон-фононного взаимодействия $R_{\text {sim }}\left(\omega_{i}\right)$ для симметричных квантовых ям с теми же значениями фононных частот, которые были использованы на рис. 1 и 2 для левого барьера. Видно, что значения параметра электрон-фононного взаимодействия оказывается больше, чем для асимметричных структур. Сам этот параметр может изменяться по вели- чине в несколько раз. При этом при малых значениях статической диэлектрической проницаемости барьеров наибольшие изменения реализуются в симметричных структурах. При больших значениях $\varepsilon_{0}$, наоборот, более значительные изменения характерны для асимметричных структур. Это позволяет подобрать оптимальный способ управления электрон-фононным взаимодействием для структур с различными барьерами.

Несколько иная ситуация реализуется в том случае, когда области фононных частот барьеров не пересекаются. На рис. 5 представлены модельные расчеты параметра $R_{\mathrm{as}}\left(\omega_{i}\right)$ для данного случая. Отметим, что вклады обеих фононных мод в электрон-фононное взаимодействие оказываются сопоставимыми по величине.

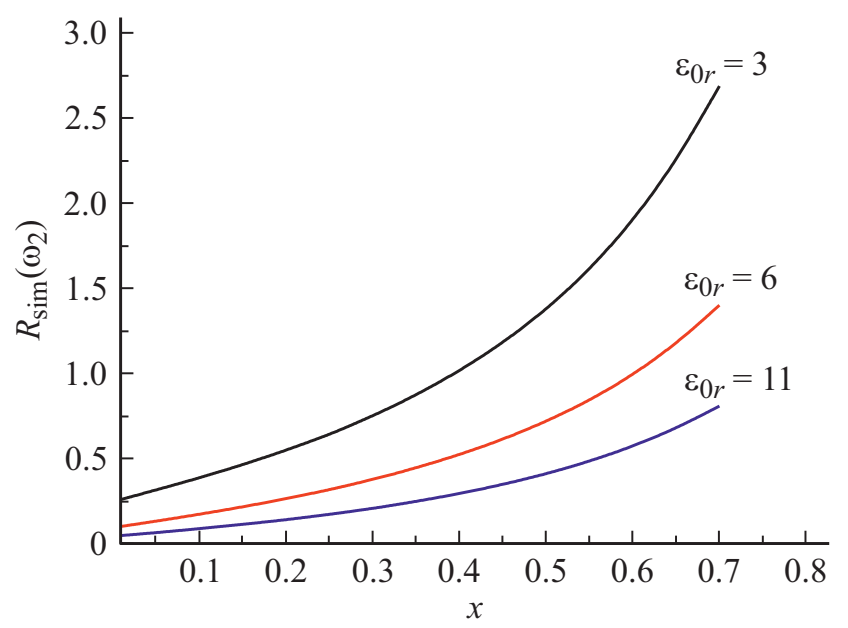

Рис. 4. Безразмерный параметр электрон-фононного взаимодействия $R_{\mathrm{sim}}\left(\omega_{2}\right)$ для одинаковых барьеров сорта $r$ с теми же модельными зависимостями частот для правого барьера, что и на рис. 1 и 2.

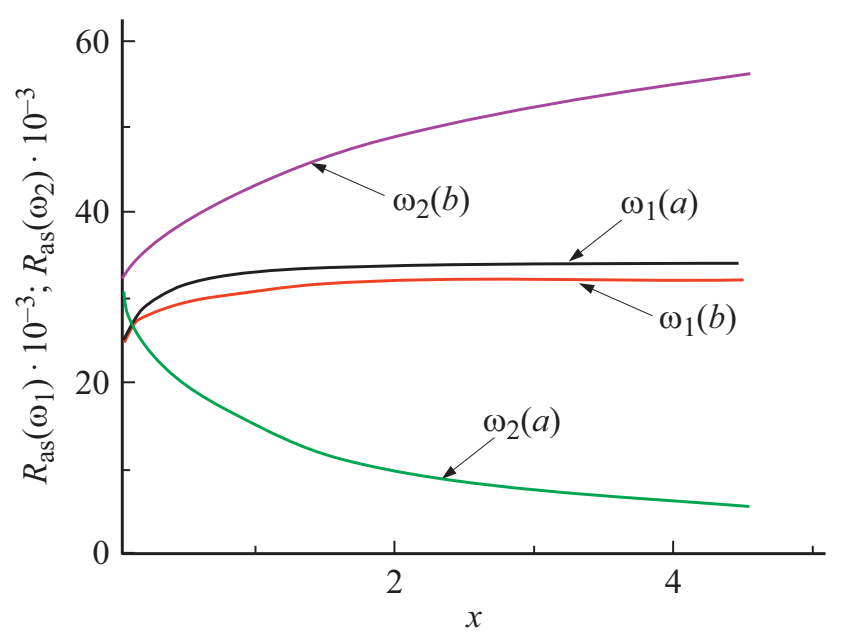

Рис. 5. Значения $R_{\text {as }}\left(\omega_{1}\right)$ и $R_{\text {as }}\left(\omega_{2}\right)$ при неперекрывающихся областях фононных частот для асимметричных барьеров $\omega_{T O, l}<\omega_{1}<\omega_{L O, l}$ и $\omega_{T O, r}<\omega_{2}<\omega_{L O, r} ; a-\omega_{L O, l}=\sqrt{2} \omega_{T O, l}$, $\omega_{T O, r}=(1.4+x) \omega_{T O, l}, \quad \omega_{L O, r}=(1.5+x) \omega_{T O, l} ; \quad b-\omega_{L O, l}=$ $=\sqrt{2} \omega_{T O, l}, \omega_{T O, r}=(1.4+x) \omega_{T O, l}, \omega_{L O, r}=(1.5+1.2 x) \omega_{T O, l}$. 
Значения параметра взаимодействия в данном случае также могут изменяться в несколько раз. Однако при близких значениях фононных частот взаимодействие для носителей в квантовой яме с симметричными барьерами по-прежнему оказывается больше.

Иная ситуация возникает при значительном различии фононных частот барьеров. В этом случае можно выполнить аналитическое сравнение параметров $R_{\mathrm{as}}\left(\omega_{i}\right)$ и $R_{\mathrm{sim}}\left(\omega_{i}\right)$. Пусть для определенности $\omega_{L O, l} \gg \omega_{L O, r}$. Тогда для интерфейсных фононов, частота которых $\omega_{1}$ близка к $\omega_{L O, l}, \beta_{l} \ll \beta_{r}$,

$$
R_{\mathrm{as}}\left(\omega_{i}\right) \approx 2 \beta_{i}\left(\omega_{1}\right)
$$

При этом при выполнении условия

$$
\varepsilon_{r, \infty}<\frac{1}{2}\left[\left(\varepsilon_{l, 0}^{2}+\varepsilon_{l, \infty}^{2}+6 \varepsilon_{l, 0} \varepsilon_{l, \infty}\right)^{1 / 2}-\left(\varepsilon_{l, 0}+\varepsilon_{l, \infty}\right)\right],
$$

получим, что

$$
R_{\mathrm{as}}\left(\omega_{i}\right)>R_{\mathrm{sim}, l}\left(\omega_{i}\right) .
$$

Это означает, что в асимметричной структуре электронфононное взаимодействие оказывается больше, чем в симметричной, где оба барьера изготовлены из материала левого барьера для исследуемой в данной работе квантовой ямы. При этом в асимметричной структуре имеется второй вклад в области относительно малых частот $\omega_{T O, r}<\omega_{2}<\omega_{L O, r}$. Для этой области, как правило, $\beta_{l}\left(\omega_{2}\right) \gg \beta_{r}\left(\omega_{2}\right), R_{\text {as }}\left(\omega_{2}\right) \approx 2 \beta_{r}\left(\omega_{2}\right)$ и условие

$$
R_{\mathrm{as}}\left(\omega_{i}\right)>R_{\mathrm{sim}, r}\left(\omega_{i}\right)
$$

реализуется при выполнении следующего соотношения

$$
\varepsilon_{l, 0}<\frac{1}{2}\left[\left(\varepsilon_{r, 0}^{2}+\varepsilon_{r, \infty}^{2}+6 \varepsilon_{r, 0} \varepsilon_{r, \infty}\right)^{1 / 2}-\left(\varepsilon_{r, 0}+\varepsilon_{r, \infty}\right)\right],
$$

Условия (17) и (20) в принципе не противоречат друг другу и могут быть реализованы одновременно. Однако если оба барьера изготовлены из материалов с высокой степенью ионности, одновременно реализовать и значительную разность фононных частот, и выполнение условий (17) и (20) оказывается невозможным. Тем не менее имеется ситуация, когда использование асимметричных барьеров создает в области квантовой ямы бо́льшую поляризацию, чем при наличии симметричных барьеров. Это возможно, если один из барьеров изготовлен из слабо полярного или неполярного материала с небольшим значением диэлектрической проницаемости. Такую структуру мы будем называть квантовой ямой с предельно асимметричными барьерами. В этом случае для получения усиленного взаимодействия достаточно выполнения только одного из неравенств - (17) или (20), в зависимости от того, какой из барьеров изготовлен из неполярного материала. Кроме того, одна из частот интерфейсных фононов обращается в нуль, и условие значительного различия частот выполняется

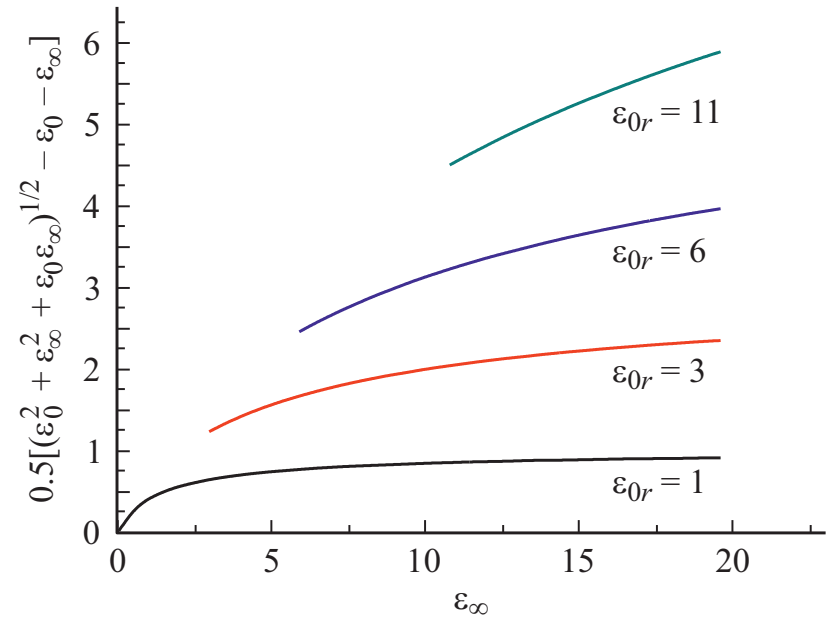

Рис. 6. Граница области усиления для предельно асимметричных барьеров при различных значениях $\varepsilon_{0 r}$.

автоматически. На рис. 6 показана граница области усиления для различных значений параметров полярного барьера. При разных значениях $\varepsilon_{0}$ эта граница меняется от $(\sqrt{2}-1) \varepsilon_{\infty}$ до $\varepsilon_{\infty}$. Для достижения усиленного взаимодействия диэлектрическая проницаемость неполярного барьера должна быть меньше этой величины.

В остальных случаях наибольшая величина электронфононного взаимодействия поляронного типа реализуется в структурах с симметричными барьерами, обладающими наибольшей степенью ионности. Следует отметить, что величина электрон-фононного взаимодействия в структурах с разными барьерами изменяется в несколько раз. Это позволяет в принципе реализовать и исследовать переход от слабого взаимодействия к сильному. Для этого материал хотя бы одного из барьеров должен обладать высокой степенью ионности, которая необходима для образования поляронов большого радиуса.

\section{4. Заключение}

Таким образом, использование для квантовой ямы несимметричных по своим диэлектрическим параметрам барьеров приводит к значительному изменению взаимодействия заряженных частиц с полярными оптическими фононами. Безразмерный параметр взаимодействия в таких структурах может изменяться в несколько раз. Это необходимо учитывать при изучении процессов релаксации горячих носителей, исследовании оптических и транспортных свойств подобных структур. Особый интерес представляет возможность усиления взаимодействия при использовании в качестве одного из барьеров неполярного материала. Это открывает дополнительные возможности для реализации условий существования поляронов большого радиуса. 


\section{Конфликт интересов}

Авторы заявляют, что у них нет конфликта интересов.

\section{Список литературы}

[1] Z. Wang, K. Reinhardt, M. Dutta, M.A. Stroscio. Phonons in bulk and low-dimensional systems - in Length-Scale Dependent Phonon Interactions; eds by S.L. Shinde and G.P. Srivastava (Springer, 2014).

[2] B.K. Ridley. Hybrid Phonons in Nanostructures (Oxford University Press, UK, 2017).

[3] A.Yu. Maslov, O.V. Proshina. In: Phonons in Low Dimensional Structures, ed. by V.N. Stavrou (IntechOpen Limited, London, UK, 2018) chap. 1, p. 3.

[4] L.V. Asryan, F.I. Zubov, N.V. Kryzhanovskaya, M.V. Maximov, A.E. Zhukov. J. Phys.: Conf. Ser., 741, 012111 (2016).

[5] S. Das, R.K. Nayak, T. Sahu, A.K. Panda. IETE Techn. Rev., 33 (1), 17 (2016).

6] W. Wang, W. Xie, Z. Deng, M. Liao. Micromachines, 10 (12), 875 (2019).

[7] А.В. Малевская, Н.А. Калюжный, Д.А. Малевский, С.А. Минтаиров, А.М. Надточий, М.В. Нахимович, Ф.Ю. Солдатенков, М.3. Шварц, В.М. Андреев. ФТП, $55(8), 699$ (2021).

[8] А.В. Чекалин, А.В. Андреева, Н.Ю. Давидюк, Н.С. Потапович, Н.А. Садчиков, В.М. Андреев, Д.А. Малевский. ЖТФ, 91 (6), 913 (2021).

[9] F. Dimroth. Phys. Status Solidi C, 3 (3), 373 (2006).

[10] Zh.I. Alferov, V.M. Andreev, M.Z. Shvarts. High-Efficient Low-Cost Photovoltaics. Recent Developments (Switzerland AG, Springer Nature, 2020) chap. 8, p. 133.

[11] F. Dimroth, T.N.D. Tibbits, M. Niemeyer, F. Predan, P. Beutel, C. Karcher, E. Oliva, G. Siefer, D. Lackner, P. Fuß-Kailuweit, A.W. Bett, R. Krause, C. Drazek, E. Guiot, J. Wasselin, A. Tauzin, T. Signamarcheix. IEEE J. Photovoltaics, 6 (1), 343 (2016).

[12] M. Mori, T. Ando. Phys. Rev. B, 40, 6175 (1989).

[13] A.Yu. Maslov, O.V. Proshina. Semiconductors, 53 (12), 1617 (2019).

Редактор А.Н. Смирнов

\section{Multiple changes in the electron-phonon interaction in quantum wells with dielectrically different barriers}

\author{
A.Yu. Maslov, O.V. Proshina \\ loffe Institute, \\ 194021 St. Petersburg, Russia
}

Abstract The specific features of the interaction of charged particles with polar optical phonons have been studied theoretically for quantum wells with the barriers that are asymmetric in their dielectric properties. It is shown that the interaction with interface phonon modes makes the greatest contribution in narrow quantum wells. The parameters of the electron-phonon interaction were found for the cases of different values of the phonon frequencies in the barrier materials. It turned out that a significant (by almost an order of magnitude) change in the parameters of the electron-phonon interaction can occur in such structures. This makes it possible, in principle, to trace the transition from weak to strong interactions in quantum wells of the same type but with different compositions of barrier materials. The conditions are found under which an enhancement of the electron-phonon interaction is possible in an asymmetric structure in comparison with a symmetric one with the barriers of the same composition. 